\title{
COMPUTER VISION BASED SMOKE DETECTION METHOD BY USING COLOUR AND OBJECT TRACKING
}

\author{
Ung Hwang and Jechang Jeong \\ Department of Electronic and Computer Engineering, \\ Hanyang University, Seoul, Korea \\ hwangung@gmail.com, jjeong@hanyang.ac.kr
}

\begin{abstract}
S
To reduce the damage from fire disaster, demand for automatic detection system by using computer vision technique is increasing. But because of false detections that are caused by various situations, it is hard to use in real. In fire detection area, to overcome this problem, the algorithm using several temporal and spatial information of object is proposed. Colour, brightness, and movement information is used to make relevant smoke detection algorithm. And continuous monitoring for several constraints is used to avoid false detections that are caused by unexpected behaviour of object. In experimental result, total 11 videos that have smoke and the other 2 videos that have no smoke is used for test performance of proposed method. It shows relevant performance for false detection and could detect almost fire in video.
\end{abstract}

\section{KEYWORDS}

Smoke Detection, Computer Vision, Object Tracking, Pattern Recognition

\section{INTRODUCTION}

In the research report of South Korea in 2014 [1], most of the fire accidents are unpredictable and approximately $10 \%$ of them is caused by unknown source. So there is need to find unexpected fire by unmanned machine for early alarming to reduce the damage of resource. In Korea, there are several researches on early detection system for automatic disaster alarming system. The reason that they were not detected early is the detection system is not fully installed in certain area, and the degree of completion of disaster focused detection algorithm is not quiet qualified to use it in real monitoring system. Various fire detection algorithms were developed to detect fire. They developed find the features of fire and smoke by various methods [2-11].

Fillips et al. proposed colour based fire detection algorithm that use difference of brightness of pixels [2]. During fire consume, fire shows wide brightness range and its brightness changed always. They use this temporal difference of pixel brightness to find fire. Chen et al. proposed algorithm that use colour range. It include equation like "Red $>$ Green $>$ Blue", and R has very high value. Basic colour of fire is good feature to find fire, because its colour is quiet strong that little hard to see in everywhere.

David C. Wyld et al. (Eds) : ITCS, JSE, SIP, ARIA, NLP - 2016

pp. 53-59, 2016. ( ) CS \& IT-CSCP 2016

DOI : 10.5121/csit.2016.60306 
When people find fire, they usually see smoke first. So smoke detect algorithm is better than fire detection algorithm for early alarming. Fire detection system usually uses both smoke and fire detection method, but smoke detection algorithm is more important for long range smoke detection, because fire usually detected by people who saw smoke that generated from the fire. By this reason, the machine monitor fire instead of human eyes also need to have more sensitive algorithm for early fire detection.

Toreyin et al. proposed smoke detection algorithm that can use temporal variation of wavelet domain. They use background of video, edge, and colour to determine the features of smoke [4]. Chen proposed a method for smoke detection algorithm by using the total number of smoke pixel that is determined by pixel differences between frames and chromatic value model of smoke [5].Yuan proposed motion based smoke detection algorithm is specially concentrated on upward moving of smoke[6]. It uses chromatic model to define smoke pixel and the accumulation of motion to distinguish between smoke and non-smoke moving object.

Smoke has similar colour and movement with non-smoke object, therefore conventional smoke detection algorithms usually have false detections. It is necessary to define more characteristic of smoke. Mainly this paper consist of two parts, one is for long distance detection, and the other one is for short range. For long distance smoke detection, Chen et al.'s method is used that count all smoke picture in the frame [4]. Smoke pixel is defined empirically in specific RGB colour range that can find all smoke in test video. For short range smoke detection, more specific moving detection algorithm is applied. These concept is deeply covered in section 2.2-2.4. Experiment results are showed in section 3.

\section{PROPOSED SMOKE DETECTION ALgORITHM}

\subsection{Pixel based smoke detection by using colour and brightness}

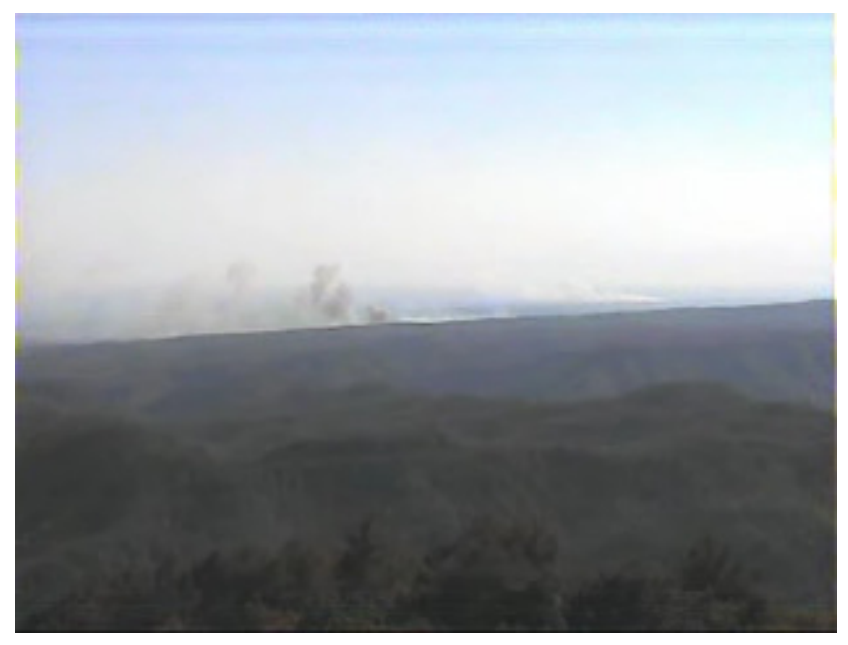

Fig.1. Long distance smoke

When fire occur, long distance smoke has a little difference between the frames even wildfire is occurred. So we have to use more sensitive detection method than short distance smoke detection. Block based algorithm that is used for fire detection is easy to analyses local area and 
classify each local area's characteristic for smoke detection. But it is hard to detect small amount of variation of frame. So in this paper we use pixel based detection algorithm to find long distance fire detection. First we find pixels that satisfy next equation:

$$
\left|Y_{x, y, n-1}-Y_{x, y, n}\right|<T H_{y}
$$

where $Y_{n}$ is brightness of pixel of $n$th frame. $T H_{y}$ is empirically selected as 20 .

In human eyes, normally smoke show achromatic colour that has similar $\mathrm{r}$, g, and $\mathrm{g}$ value. But in videos, smoke does not has achromatic colour always. So smoke colour model is designed to can include all kinds of smoke colours in videos. The equation is shown in eq. (2). Smoke normally shows achromatic colours, but these kinds of colour are easily found on everywhere.

Black car, grey shirt and white wall are example of potential smoke candidates in the view of smoke colour. So colour based smoke detection is not efficient itself, So motion or appearance based detections have proposed definition by colour more than fire, monitored behaviours of smoke is used to detect fire in this paper.

$$
\begin{gathered}
\left|\mathrm{R}_{\mathrm{x}, \mathrm{y}}-\mathrm{G}_{\mathrm{x}, \mathrm{y}}\right|<\mathrm{TH}_{\mathrm{CS}} \\
\left|\mathrm{G}_{\mathrm{x}, \mathrm{y}}-\mathrm{B}_{\mathrm{x}, \mathrm{y}}\right|<\mathrm{TH}_{\mathrm{CS}}+80 \\
\left|\mathrm{~B}_{\mathrm{x}, \mathrm{y}}-\mathrm{R}_{\mathrm{x}, \mathrm{y}}\right|<\mathrm{TH}_{\mathrm{CS}}+80
\end{gathered}
$$

\subsection{Object based smoke detection}

To partition each object, labelling algorithm distinguish each object is used. And for relevant partitioning, open and close filter which use min and max filter are used. The open filter is the filter which uses min filter after max filter. The close filter is opposite. By using open filter, it is easy to remove noise pixels that has smoke feature in the frame without big data losses of original shape. By using close filter, divided smoke pixel can be joined as one object. Fig 2 shows open and close filter reduced noise and centralized and organized smoke. By this partitioning object method, groundwork for using method of Section 2.3 is ready.

\subsection{Behaviour based smoke detection}

For long distance detection, there are not many objects that cause false detection. But for short distance detection, there are many things to exclude to find the real smoke. One of major cause is smoke has colour that can be easily found everywhere, so colour based smoke detection method cannot occupy big portion of smoke detection algorithm.

Smoke shows uncommon behaviour that becomes large and goes upward. These kinds of moving are used to detect smoke in [5-6]. These principles of smoke detection are very helpful to find smoke and this concept is also applied in the proposed algorithm. But if there is object that has same colour and do same move (going upward in frame), that object is easy to occur false detection. To avoid this, in this proposed algorithm, there are some constraints that can remove false candidate of smoke by finding objects that have not smoke behaviour. 


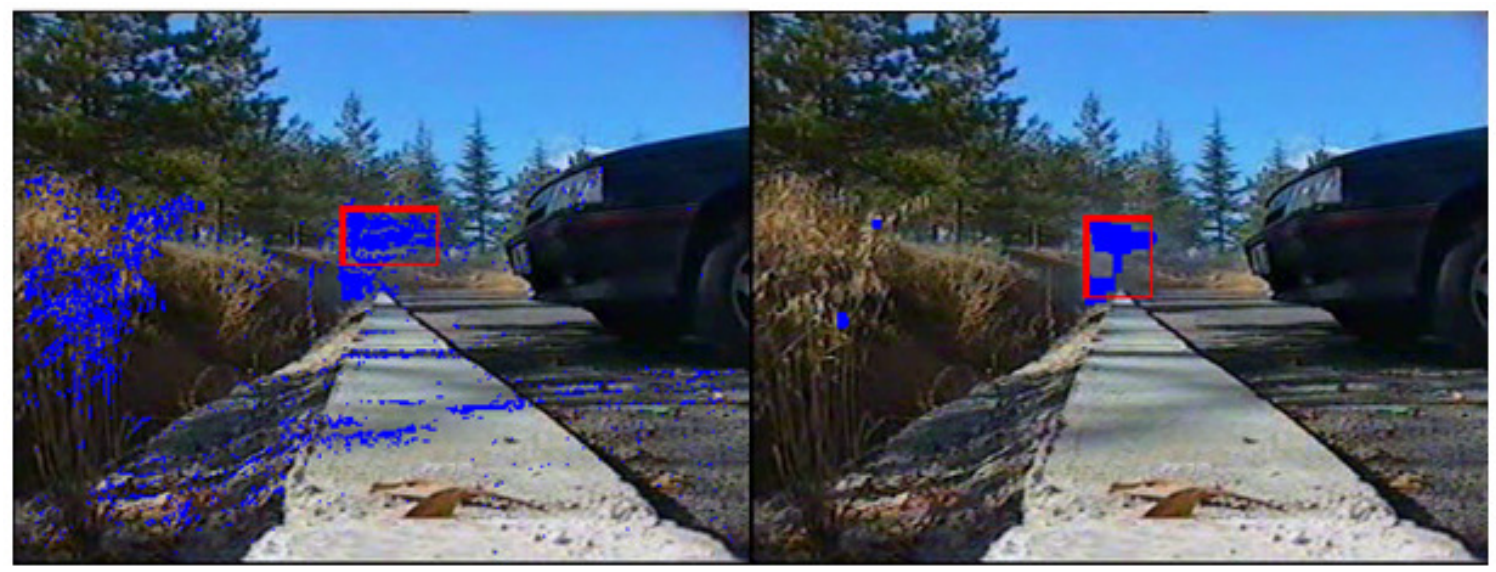

(a)

(b)

Fig 2. (a) Smoke pixel image (b) Filtered image by using open and close filter

\subsubsection{Natural smoke attribute}

Smoke is diffused well because it has high temperature, and burning materiel makes smoke continually. In [1], they concluded that smoke is in the video if smoke pixel in the image increase continually for a moment. In this paper, long distance smoke detection use this method. And smoke pixels have similar brightness between near pixel. Its colour is not changed immediately like fire (fire shows various colour) in the small region. So if some object has various colour in a same object and each near pixels brightness are not similar each other, those object is not consider as same object. In [2], they use this attribute to find a smoke.

\subsubsection{Estimate smoke movement by using camera position}

Usually to find smoke, camera is installed in higher position than monitored area. So if some object is coming to under camera, it become bigger in frame, but its position become lower continually. So we can assume it is not smoke, but another object.

\subsubsection{Position of fire}

We assume fire is located in camera frame or under camera frame. So the object that comes from side of frame is not considered as smoke. And position that smoke appear first is should be remain for a moment. Because we assume fire become bigger and bigger, position that smoke appear first is should be maintained and not moved if smoke is being generated.

\subsubsection{Sudden changing}

We assume to use 15-30 f/s camera for fire detection. This is very short time to make big difference in frame. So we can make threshold to limit the size changing of object or moving distance of object. Normal object cannot go far within 1/30 second and even smoke cannot bigger than specific amount. So unacceptable cases are considered as error and ignored. 


\subsection{Final Decision}

In the final decision, alarm can occur if all condition is satisfied. The object continually become bigger for a second, and that satisfy rule of 2.3 is decided as smoke object and alarm occur immediately.

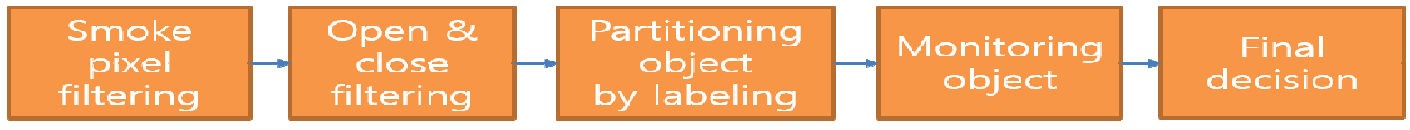

Fig 3. Block diagram of proposed algorithm

\section{EXPERIMENTAL RESULT}

In Fig. 4, there are 11 videos that have smoke and 4 videos that do not have smoke. The main causes of false detection in this proposed algorithm is the object in front of smoke. Because the proposed algorithm uses object based detection, if shape of smoke is ruined then proposed algorithm can makes error. Usually smoke is on the sky and there is nothing between smoke and detector, so camera installed in proper position, it can overcome. The other false detection is caused by two object that has similar colour. If they meet and divide frequently, the proposed algorithm cannot distinguish there two object and they become one object (become bigger than before). The proposed algorithm only make false detections for these situation, so it will be the future work.

Table 1. Experimental result.

\begin{tabular}{|c|c|c|c|}
\hline Video & Detection & Comment & $\begin{array}{c}\text { Number of false } \\
\text { detection }\end{array}$ \\
\hline Smoke 1 & True & - & 0 \\
\hline Smoke 2 & True & - & 0 \\
\hline Smoke 3 & True & - & 0 \\
\hline Smoke 4 & True & - & 0 \\
\hline Smoke 4 & True & - & 0 \\
\hline Smoke 5 & True & - & 0 \\
\hline Smoke 6 & True & - & 0 \\
\hline Smoke 7 & False & $\begin{array}{l}\text { Cannot detect smoke in the sky, } \\
\text { (Too little amount of smoke, similar } \\
\text { colour with sky) }\end{array}$ & 0 \\
\hline Smoke 8 & False & $\begin{array}{c}\text { Smoke is divided two because of an } \\
\text { object in front of smoke }\end{array}$ & 0 \\
\hline Smoke 9 & True & - & 0 \\
\hline Smoke 10 & True & - & 0 \\
\hline Smoke 11 & True & - & 0 \\
\hline Non-smoke 1 & True & - & 0 \\
\hline Non-smoke 2 & True & - & 0 \\
\hline Non-smoke 3 & False & $\begin{array}{l}\text { Smoke divide two because of an object in } \\
\text { front of smoke }\end{array}$ & 2 \\
\hline Non-smoke 4 & False & $\begin{array}{c}\text { Two object shows similar characteristics. } \\
\text { They united and divide frequently. }\end{array}$ & $\begin{array}{c}5 \\
\text { (long size video) }\end{array}$ \\
\hline
\end{tabular}


Computer Science \& Information Technology (CS \& IT)

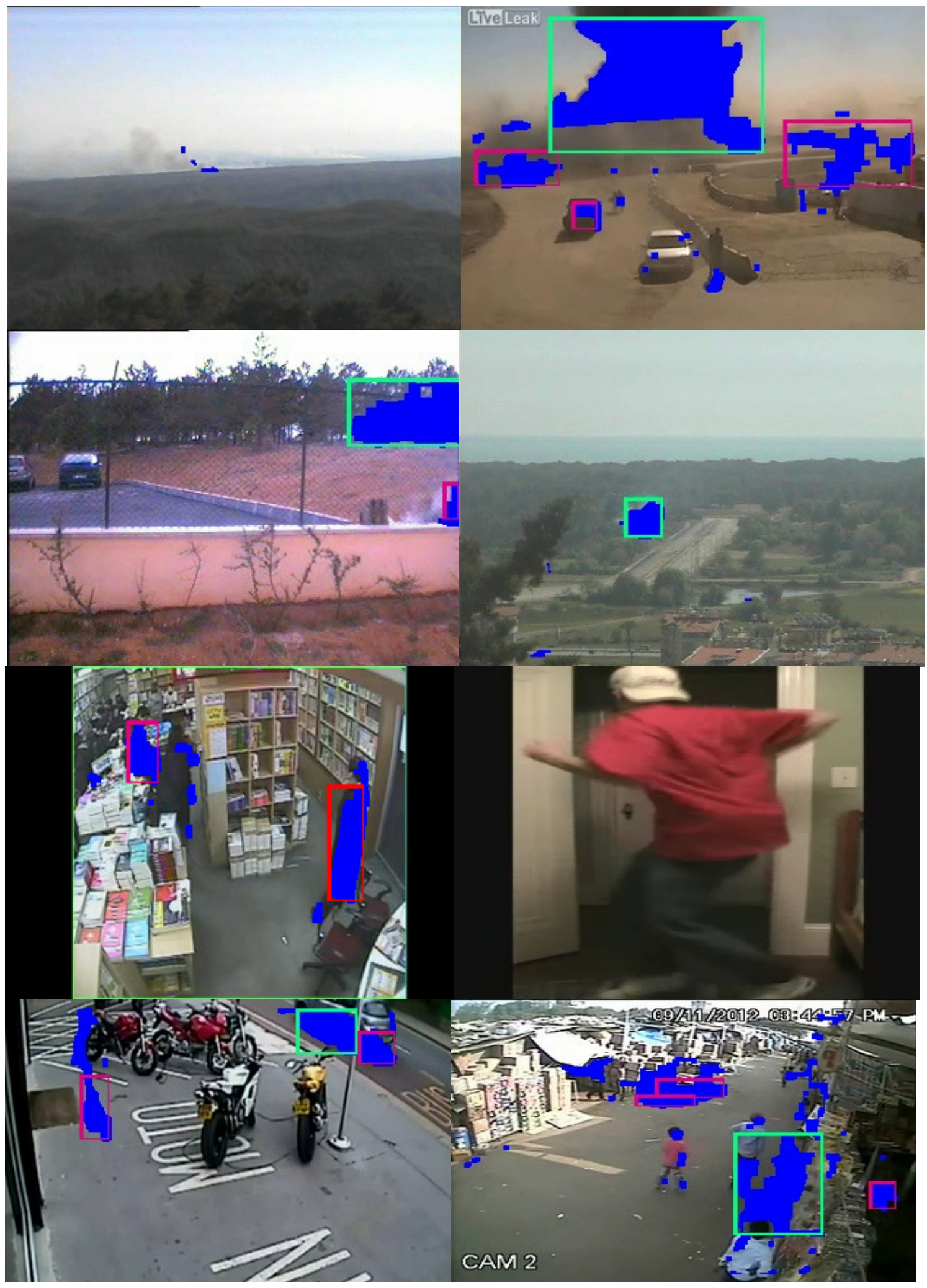

Fig 4. Experimental Result - First 4 Video has smoke, last 4 video do not has smoke 


\section{CONCLUSIONS}

There are many methods to detect fire for early detection that can reduce damage of accident. In this paper, smoke detection method is proposed to decrease the false detection for better use in real. To reduce the false detection rate, we use labelling and object tracking that can monitor behaviour of each object. By monitored data, smoke object is determined as smoke when all movement and characteristic are satisfied to decide. By using proposed algorithm, false detection rate is lowered than before, however in some unexpected condition there are still some errors. If we can use better object tracking method and computing power is enough to use this, it can be covered in future work.

\section{ACKNOWLEDGEMENTS}

This work was supported by Institute for Information \& communications Technology Promotion(IITP) grant funded by the Korea government(MSIP) (No.2015-B0101-15-1377,Development of 16-channel Multi-viewer, H/W Encoder, and Transcoder Systems for 4K UHD Broadcasting)

\section{REFERENCES}

[1] Fire report in Korea Oct / 2014, http://usafe.namwon.go.kr/boardread_ifr.asp?g=1\&idx=754\&cnt=9.

[2] W. Phillips Iii, M. Shah, and N. da Vitoria Lobo, "Flame recognition in video," Pattern recognition letters, vol. 23, pp. 319-327, 2002.

[3] T.-H. Chen, P.-H. Wu, and Y.-C. Chiou, "An early fire-detection method based on image processing," in Image Processing, 2004. ICIP'04.2004 International Conference on, 2004, pp. 1707-1710

[4] B. U. Toreyin, Y. Dedeoglu, and A. E. Cetin, "Contour based smoke detection in video using wavelets," European Signal Processing Conference, pp. 123-128, 2006.

[5] T.-H. Chen, Y.-H. Yin, S.-F. Huang, and Y.-T. Ye, "The smoke detection for early fire-alarming system based on video processing," in Intelligent Information Hiding and Multimedia Signal Processing, 2006. IIH-MSP'06. International Conference on, 2006.

[6] F. Yuan, "A fast accumulative motion orientation model based on integral image for video smoke detection," Pattern Recognition Letters, vol. 29, pp. 925-932, 5/1/ 2008.

[7] Y. Hakan Habiboglu, O. Gunay, and A. Enis Cetin, "Real-time wildfire detection using correlation descriptors," in Signal Processing Conference, 2011 19th European, 2011, pp. 894-898.

[8] A. Benazza-Benyahia, N. Hamouda, F. Tlili, and S. Ouerghi, "Early smoke detection in forest areas from DCT based compressed video," in European Signal Processing Conference, 2012, pp. 2752-2756.

[9] M. Stula, D. Krstinic, and L. Seric, "Intelligent forest fire monitoring system," Information Systems Frontiers, vol. 14, pp. 725-739, 2012/07/01 2012

[10] B. Ko, J. Park, and J.-Y. Nam, "Spatiotemporal bag-of-features for early wildfire smoke detection," Image and Vision Computing, vol. 31, pp. 786-795, 2013

[11] D. Krstinić, D. Stipaničev, and T. Jakovčević, "Histogram-based smoke segmentation in forest fire detection system," Information Technology and Control, vol. 38, 2015. 